\title{
The Muon Spectrometer Barrel Level-1 Trigger of the ATLAS Experiment at LHC
}

\author{
A. Aloisio, G. Carlino, F. Conventi, R. de Asmundis, V. Izzo, A. Migliaccio \\ G. Ciapetti, A. di Mattia, C. Luci, L. Luminari, A. Nisati, F. Pastore, E. Petrolo, R. Vari, \\ S. Veneziano
}

A. Salamon

\begin{abstract}
The proton-proton beam crossing at the LHC accelerator at CERN will have a rate of $40 \mathrm{MHz}$ at the project luminosity. The ATLAS Trigger System has been designed in three levels in order to select only interesting physics events reducing from that rate of $\mathbf{4 0}$ MHz to the foreseen storage rate of about $200 \mathrm{~Hz}$. The First Level reduces the output rate to about $100 \mathrm{kHz}$. The ATLAS Muon Spectrometer has been designed to perform stand-alone triggering and measurement of muon transverse momentum up to $1 \mathrm{TeV} / \mathrm{c}$ with good resolution (from $3 \%$ at $10 \mathrm{GeV} / \mathrm{c}$ up to $10 \%$ at $1 \mathrm{TeV} / \mathrm{c}$ ). In the Barrel region of the Muon Spectrometer the Level1 trigger is given by means of three layers of Resistive Plate Chamber detectors (RPC): a gaseous detector working in avalanche mode composed by two plates of high-resistivity bakelite and two orthogonal planes of read-out strips. The logic of the Level-1 barrel muon trigger is based on the search of patterns of RPC hits in the three layers consistent with a high transverse momentum muon track originated from the interaction vertex. The associated trigger electronics is based on dedicated processors, the Coincidence Matrix boards, performing space coincidences and time gates and providing the RPC readout as well. A detailed simulation of the ATLAS Experiment and of both the hardware components and the logic of the Level-1 Muon Trigger in the barrel of the Muon Spectrometer has been performed. This simulation has been used not only to evaluate the performances of the system but also to define the hardware set-up such as the cabling of both the trigger detectors and the trigger electronics modules. A description of both the Level-1 Muon Trigger system in the barrel and the RPC detectors, with their cosmic rays quality tests, will be presented together with the trigger performances and rates calculations evaluated for muons over a wide range of $p_{T}$ and preliminary studies on the impact of accidental triggers due to low energy background particles in the experimental area.
\end{abstract}

A. Aloisio, G. Carlino, F. Conventi, R. de Asmundis, V. Izzo, A. Migliaccio are with the INFN-Napoli and also with the University of Naples "Federico II" Italy (corresponding author is Riccardo de Asmundis, INFN - Complesso di Monte S. Angelo, via Cintia 80126 NAPOLI; Italy. Riccardo.deasmundis@na.infn.it, phone +39081676179 , fax +39081676246 )

G. Ciapetti, A. di Mattia, C. Luci, L. Luminari, A. Nisati, F. Pastore, E. Petrolo, R. Vari, S. Veneziano are with the University of Rome "La Sapienza" and INFN-Roma, Italy.

A. Salamon was with the University of Rome "La Sapienza" and now is affiliated to University of Rome 2 "Tor Vergata" and INFN Roma 2, Roma, Italy.

\section{I. - INTRODUCTION}

The proton-proton Collider LHC at CERN, currently under construction, has a design luminosity of $\mathcal{L}=$ $10^{34} \mathrm{~cm}^{-2} \mathrm{~s}^{-1}$ with a centre of mass energy of $14 \mathrm{TeV}$ and a bunch crossing rate of $40 \mathrm{MHz}$. ATLAS is one of the four experiments under construction on LHC. It is characterised by an inner detector consisting of several layers of silicon pixels, strips, straw tubes, all inside a 2 $\mathrm{T}$ solenoid magnetic field. An electromagnetic calorimeter in liquid argon and an hadronic tile calorimeter (made of iron and plastic scintillators), surround the central detector. Similar configurations (but in liquid argon for hadronic part too) are provided for end cap detector. The Muon spectrometer surrounds this inner detector.

\section{II. -THE MUON SPECTROMETER}

The Muon spectrometer measures the transverse momentum $\mathrm{p}_{\mathrm{T}}$ of muons both in the barrel region and in the end cap; for this a stand alone trigger is provided to identify the muons coming from Interaction Point (IP) and in time with bunch crossing. The spectrometer is based on precision detectors: the Monitored Drift Tubes (MDTs) which track the muons in a toroidal magnetic field for the barrel, and Cathode Strip Chambers for the end cap. Three multi-layer stations of MDT give, after reconstruction process, three main geometrical points along the track, from where a sagitta and consequently the transverse momentum $\mathrm{p}_{\mathrm{T}}$ can be calculated. Each multi-layer is composed by three or four planes of tubes, so that the position resolution can be increased with respect to the single wire resolution and left-right ambiguity can be resolved. Trigger functionality is carried on by the RPCs detectors for the barrel and the TGC (Thin Gap Chambers) for the end cap.

\section{III. - THE TRIGGER}

The ATLAS trigger is organised in three levels. The first level must apply a rate reduction from the $40 \mathrm{MHz}$ of bunch crossing of LHC to a final value of $75-100$ $\mathrm{kHz}$ of accepted events.

At the final luminosity of $10^{34}$ in the units $\mathrm{cm}^{-2} \mathrm{~s}^{-1}$ we expect a ratio of about 23 in the production of minimum bias events with respect to hard interactions. 
The latter are the only interesting events for physics. Transverse momentum $\mathrm{p}_{\mathrm{T}}$ is a main signature for these events: they are characterized by a value of $\mathrm{p}_{\mathrm{T}}$ greater than few $\mathrm{GeV} / \mathrm{c}$, allowing the introduction of a cut in $\mathrm{p}_{\mathrm{T}}$. The level 1 trigger uses information from the calorimeter and muon detector only. "Coarse" data from Calorimeters and Muon spectrometer are combined into the Central Trigger Processor for final decision. Tracking from both central detectors and fine data from MDTs are used from second level on. Figure 1 shows a sketch of the structure of the first level of the trigger. Trigger processing is logically sketched by the two grey boxes (for Calorimeter and Muon), while final trigger information are sent to the Central Trigger Processor for successive stages and for Data Acquisition (DAQ) firing.

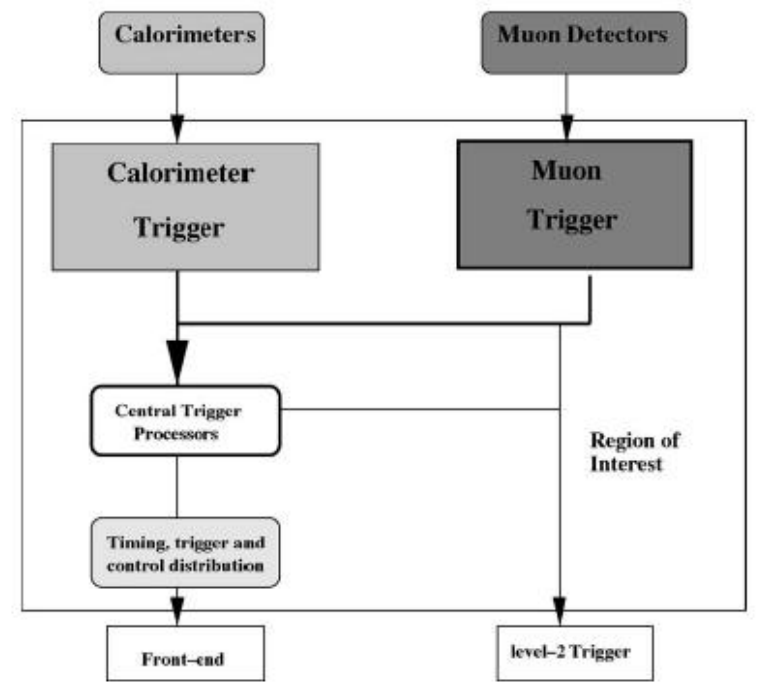

Figure 1: the structural logic of the first level Trigger of ATLAS

Note that Muon trigger produces Region of Interest (RoI) too: these information are then used by second level trigger to deeply inspect the events for their acceptation. Latency times for different levels are 2.5 $\mu \mathrm{s}$ for the first, $10 \mathrm{~ms}$ and $1 \mathrm{~s}$ for second and third respectively. Level 2 trigger and Event Filter ( $3^{\text {rd }}$ level) are software filters written in $\mathrm{C}++$. Detailed descriptions can be found in $[1,2]$.

\section{IV. - THE MUON TRIGGER IN THE BARREL REGION}

The RPC are used as trigger detectors in the first level. They are characterized by a very high time resolution (about $1.5 \mathrm{~ns}$ ) and a spatial resolution of $1 \mathrm{~cm}$ that allows use of a fast information about track position to determine if the detected muons come from IP in time with a bunch crossing. The first level, and thus the muon trigger, must act in a maximum latency time of 2 $\mu \mathrm{s}$ (2.5 $\mu \mathrm{s}$ with a certain "contingency"). The RPCs cover a surface of 3,500 square meters and are installed as a doublet on the central MDT station and a singlet on the outer one. Each RPC is composed by two sensitive layers of gas detectors (working in avalanche mode with a gas mixture of tetrafluorethane, isobuthane and $\mathrm{SF}_{6}$ ), each of them able to read both pseudorapidity $\eta$ and azimuthal $\phi$ coordinates with a resolution of about $1 \mathrm{~cm}$. Figure 2 is a sketch of the main structure of the RPCs used in the muon trigger. Note that here only one sensitive layer is shown.

The signals from RPC detector are amplified, discriminated and digitally shaped on-detector. Then they are sent to a Coincidence Matrix (CM) board which contains a CM chip. This chip performs almost all of the functions needed for the trigger algorithm and also for the read-out of the strip. It implements the low$\mathrm{p}_{\mathrm{T}}$ and the high- $\mathrm{p}_{\mathrm{T}}$ trigger algorithms, depending on their positions on the spectrometer: it aligns in time the input signals, performs the coincidence and makes the $\mathrm{p}_{\mathrm{T}}$ cut on the different thresholds. It also contains the level-1 latency pipeline memory and derandomiser buffer. The CM board produces an output pattern containing the low- $\mathrm{p}_{\mathrm{T}}$ (or the high- $\mathrm{p}_{\mathrm{T}}$ ) trigger results and the hit list for each pair of RPC doublets in the $\eta$ or $\phi$ projection.

The information of two adjacent CM boards in the $\eta$ projection, and the corresponding information of the two CM boards in the $\phi$ projection, are combined together in the low- $\mathrm{p}_{\mathrm{T}}$ Pad Logic (Pad) board. The Pad boards are mounted on top of the RPC2 detectors for low- $\mathrm{p}_{\mathrm{T}}$ determination and equivalently on RPC3 detectors for high $\mathrm{p}_{\mathrm{T}}$. Low- $\mathrm{p}_{\mathrm{T}}$ trigger results and the associated RoI are transferred synchronously at 40 $\mathrm{MHz}$, to the corresponding high- $\mathrm{p}_{\mathrm{T}}$ Pad board that collects the overall result.

RPCs and MDTs locations are shown in Figure 3, where some "triggering" tracks are also drawn. Thresholds values for $\mathrm{p}_{\mathrm{T}}$ are 6,8 and $10 \mathrm{GeV} / \mathrm{c}$ for low algorithm and 20 to $40 \mathrm{GeV} / \mathrm{c}$ for high- $\mathrm{p}_{\mathrm{T}}$ one.

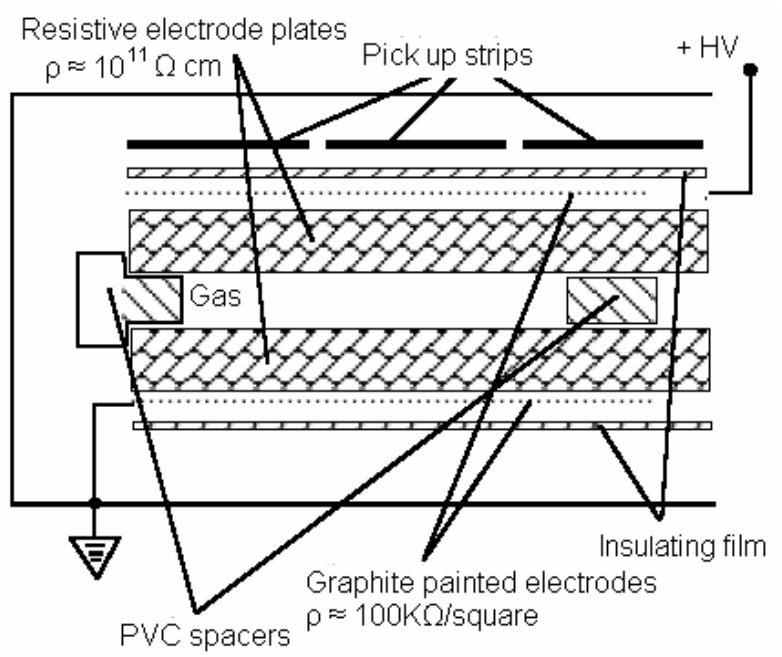

Figure 2: structure of RPC detector. One sensitive layer only is shown.

To take into account all the different coordinates, thresholds and geometry of the whole apparatus, a total of more than 3,000 CMs have been calculated using a specific sample of single muon tracks. 


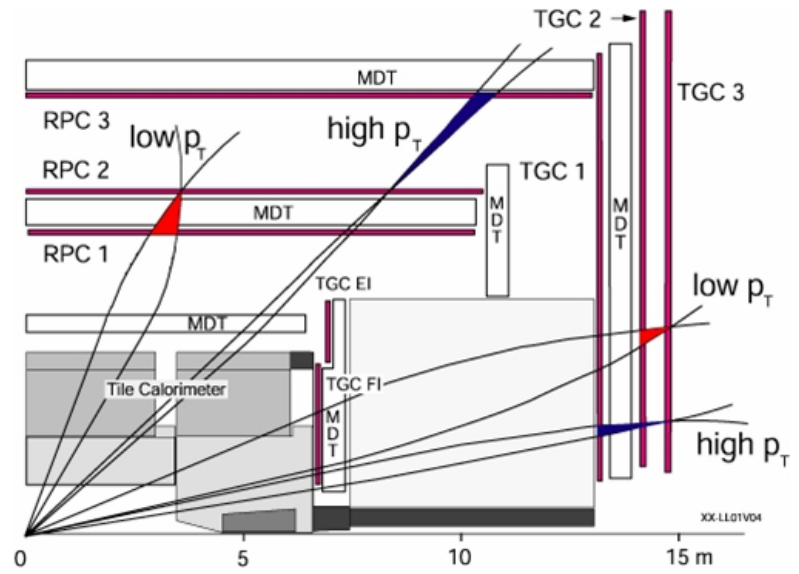

Figure 3: typical muon tracks generating triggers in both barrel and endcap regions.

The global trigger logic is steered by hits in RPC2 layer, also called the pivot plane. In such a case RPC1, the confirmation plane, is searched for a coincident hit within the relative $\mathrm{CM}$, to indicate a track pointing to the vertex. The higher is the muon $\mathrm{p}_{\mathrm{T}}$, the smaller is the spread of the track, in the sense it will be expected within a lower number of strips in the confirmation plane with respect to the projection of the pivot hit. This spread is mostly of the order of 3-6 RPC strips, equivalent to 9 to $18 \mathrm{~cm}$ in length. The low- $\mathrm{p}_{\mathrm{T}}$ trigger is generated in case of a track within this CM limits and in time with the bunch crossing signal. The same operation but with RPC3 is performed by the high- $\mathrm{p}_{\mathrm{T}}$ algorithm: in that case, logic is steered by the already asserted low- $\mathrm{p}_{\mathrm{T}}$ trigger which acts in the place of the pivot plane. The logic is applied with a $3 / 4$ majority for low- $\mathrm{p}_{\mathrm{T}}$ and $1 / 2$ majority for high $\mathrm{p}_{\mathrm{T}}$; majority indicates that, in the first case, 3 out of 4 RPC sensitive layers must produce an hit to give a low- $\mathrm{p}_{\mathrm{T}}$ trigger; 1 out of 2 in the second case (for high $\mathrm{p}_{\mathrm{T}}$ ).

The whole spectrometer is divided in $\phi$ coordinate into 64 logical sectors, 32 on each side of detector (i.e. positive and negative $\eta$ coordinate).

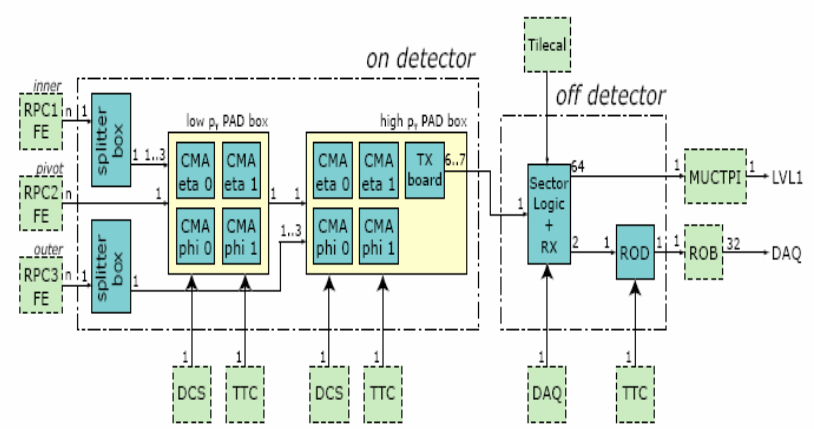

Figure 4: the Muon Trigger data acquisition system. Note, on the right (off detector), the presence of Sector Logic (SL), Read Out Buffers (ROB) and Central Trigger Processor (MUCTPI)

Data from PADs are sent via optical links to read out system, based on appropriate receivers, Read Out
Drivers (ROD), Sector Logic, Read Out Buffers and Central Trigger Processors.

See Figure 4 for the organization of DAQ system, where "on detector" and "off detector" equipments are shown. Small sectors receive data from seven PAD logics, while large sectors from six ("Small" sectors are the ones installed ON the toroid magnet coils, while "Large" sectors are the ones between coils. Using the ATLAS sectors classification, all even sectors are small and all odd sectors are large). See $[3,4]$ for details on Pads and Sector logic.

\section{V. - THE TESTS ON RPC DETECTORS}

The RPCs detectors have been subjected to different quality tests and certification before coming to the experiment. Some first Quality Control tests have been performed at production sites on specific stations in Naples, Lecce and Rome. An ageing test has been performed at CERN, X5 site, in an high radiation environment simulating several times the background conditions foreseen in the ATLAS cavern. A total of 8 years equivalent have been integrated (with a safety factor of 5) and the RPCs have shown a good tolerance to ageing: efficiency and gap currents are stable, while bakelite resistivity has shown an increment due to ageing but still within limits to ensure good functioning of detectors.

Finally an integration test of all systems together (tracking, calorimeters, MDTs, RPCs and detectors for forward region) have been performed at CERN in H8 area on Summer 2004: in this test the RPCs have been operating in a final configuration, with the trigger processors installed and used to determine trigger decision of the muons coming from test beam.

Appropriate runs to 25 ns of inter-bunch time (corresponding to $40 \mathrm{MHz}$ of bunch rate) have been executed according to the project design. References for these subjects can be found in $[5,6,7,8]$.

\section{VI. - THE TRIGGER SIMULATIONS AND THEIR RESULTS}

Some specific simulation has been performed on both the ATLAS detectors and the trigger logic. Simulating these processes is mandatory not only to estimate the trigger performances but also to optimise design parameters. The simulation includes all passive materials and sensitive parts of detectors, electronic response and trigger hardware and logic. The simulation package is written in $\mathrm{C}++$ and runs under the official framework of ATHENA. Geant 4 package is used for description of the detector geometry [9].

The Coincidence Matrix, Pad, and Sector logic are all included in the simulation. More than 3000 CM have been defined on both projections $\eta$ and $\phi$ and for the three different momentum thresholds; to do this we used a sample of single muons with transverse momenta equal to the threshold of interest: for low- $\mathrm{p}_{\mathrm{T}}$ 6, 8 and $10 \mathrm{GeV} / \mathrm{c}$ and for high- $\mathrm{p}_{\mathrm{T}} 20$ and $40 \mathrm{GeV} / \mathrm{c}$. The format required by the hardware has been considered so that the data flow towards ROD drivers has been completely simulated. The data structure includes the CM boards fragment (which includes the 
RPC strips fired), the trigger output and the highest threshold satisfied by the trigger logic.

The sample was composed by $10^{6}$ single muons events in the $\mathrm{p}_{\mathrm{T}}$ range from 3 to $50 \mathrm{GeV} / \mathrm{c}$. the simulation results can be summarised with the following items:

- Calculation of geometrical acceptance

- Estimation of trigger efficiency

- Effect of background in creating fake triggers and on trigger efficiency

Figure 5 shows the trigger efficiency for low- $\mathrm{p}_{\mathrm{T}}$ tracks, calculated including all sectors. Special sectors as "feet" chambers (RPC Chambers covering the interspace in the feet of the apparatus; feet represent a big obstacle to the trigger coverage, and is locally resolved by using RPC with special shapes) and lower part of the apparatus, since they are subject to a lower efficiency, have been excluded form the estimation of the global one, but independently calculated.

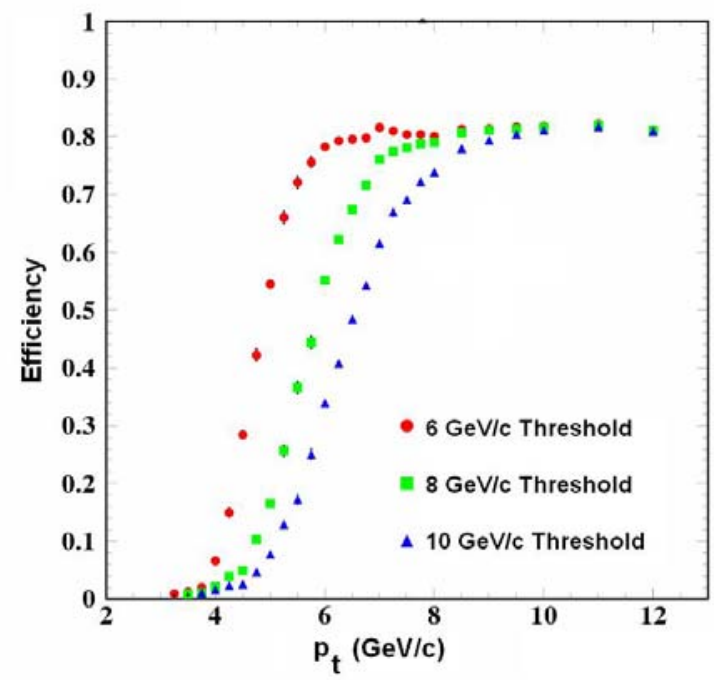

Figure 5: trigger efficiency for low- $\mathrm{p}_{\mathrm{T}}$ algorithm

Observe the slope of all efficiency curves, especially that for $6 \mathrm{GeV} / \mathrm{c}$ threshold: design parameters as Coincidence Matrixes have been adjusted to reduce as efficiently as possible the trigger rate of muons having a transverse momenta lower than the desired threshold. This is very important to avoid excessive rate due to muons with low momenta, which are basically produced in the decays in flight of the $\mathrm{K}$ and $\pi$ mesons, whose processes show an high cross section at $\mathrm{p}_{\mathrm{T}}$ below $6 \mathrm{GeV} / \mathrm{c}$. See

Figure 8 for typical cross sections of processes as a function of $\mathrm{p}_{\mathrm{T}}$. It is interesting to compare these results with the previous ones in [2].

Coming back to the special part of the apparatus, you can observe in Figure 6, the trigger inefficiency plotted as a global $\eta-\phi$ projection. Note, in greyed rectangles, special equipments of the apparatus, like support structures (3), elevator hole (2), “ $\eta-0$ ” crack (4), and ribs in small sectors (1).

Similar procedure is used to obtain global efficiency for high $\mathrm{p}_{\mathrm{T}}$ whose results are shown in Figure 7.

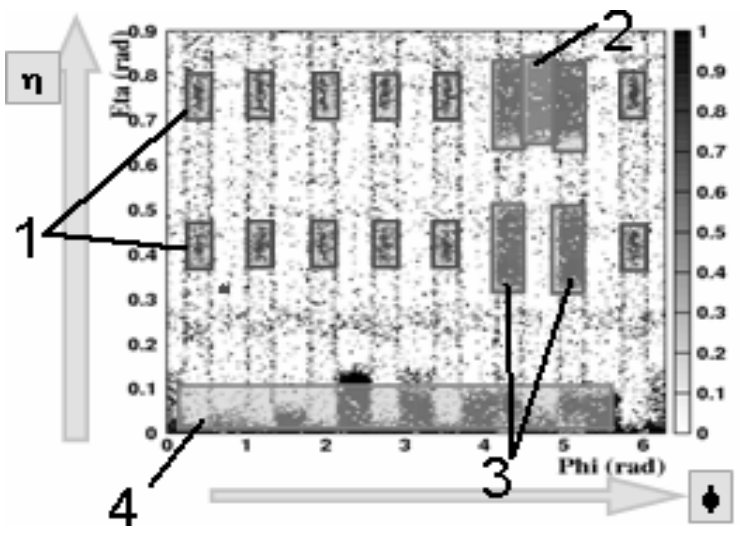

Figure 6: projection of geometrical acceptance on $\eta$ and $\phi$ coordinates. Black points indicate inefficient events, i.e. simulated tracks not detected by Trigger due to geometrical inefficiency.

Although the $40 \mathrm{GeV} / \mathrm{c}$ efficiency curve shows in the lower part quite a smooth decrease with a significant efficiency even below the $\mathrm{p}_{\mathrm{T}}$ threshold, this is perfectly acceptable since strongly above typical momenta of minimum bias events. Do not forget that trigger must only indicate interesting muon tracks, while it is a goal of precision chambers (MDT) to calculate the exact momentum.

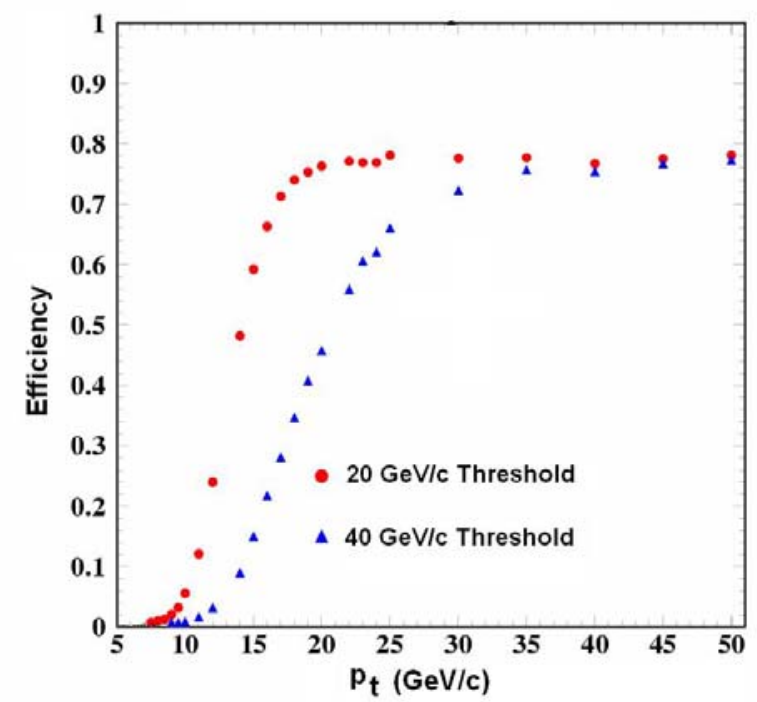

Figure 7: global efficiency for high $\mathrm{p}_{\mathrm{T}}$ algorithm

The trigger rates are calculated by the convolution of the efficiencies with the cross section extracted from Monte Carlo simulation of the physical processes that give rise to muons in the detector. This procedure has been performed for prompt single-muons production, from $\mathrm{b}$ and $\mathrm{c}$ hadrons and from $\mathrm{W}$ and $\mathrm{Z}$ decays, and for decays in flight to muons of the $\pi$ and K mesons. The cross sections, shown in 
Figure 8 as a function of $\mathrm{p}_{\mathrm{T}}$, are dominated by semileptonic decays of $b$ and $c$ hadron for $\mathrm{p}_{\mathrm{T}}>8 \mathrm{GeV} / \mathrm{c}$ whereas for $\mathrm{p}_{\mathrm{T}}<8$ the dominant source of muons, and thus of the trigger rate, are the $\mathrm{K} / \pi$ decays in flight. The inclusive muon cross section at LHC have been calculated using the Monte Carlo program Pythia (version 5.7) [10], while the secondary muons originating from $\mathrm{K} / \pi$ decays using the DPMJET MC program [11]. Trigger rates, calculated at the nominal luminosity, are shown in Table 1.

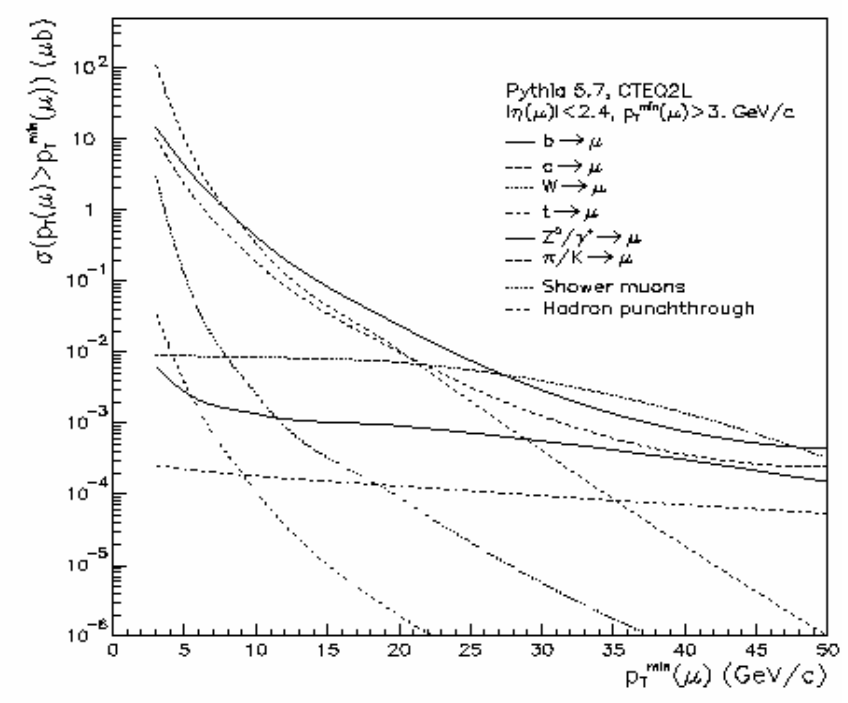

Figure 8: inclusive muon cross-section as a function of $\mathbf{p}_{\mathbf{T}}$ (from Paule Anne-Mary Eerola "The inclusive muon cross-section in ATLAS” ATL-PHYS-98-120, CERN, 1998)

\begin{tabular}{lll}
\hline Muon & Low pT: $6 \mathrm{GeV} / \mathrm{c}$ & $\mathrm{High} p T: \quad 20$ \\
sources & threshold & $\mathrm{GeV} / \mathrm{c}$ threshold \\
$\pi / K$ & 7100 & 680 \\
$\mathrm{~b}$ & 1400 & 500 \\
$\mathrm{C}$ & 800 & 210 \\
$\mathrm{~W}$ & 3 & 26 \\
$\mathrm{t}$ & negligible & negligible \\
(expected rates in $\mathrm{Hz}$ ) & & \\
\hline
\end{tabular}

Table 1: trigger rates in $\mathrm{Hz}$ from prompt and secondary muon production

Background effects have been finally estimated as source of possible reduction in trigger efficiency. As you can see in Figure 9 comparison between efficiency without and with background (doubled with respect to expected value in the LHC cavern) shows a similar behaviour, where plot points can be definitely confused. Plots with background in the figure stays beyond the ones without it and the points that are not superimposed are indicated with a specific marker (see legend on the plot itself).

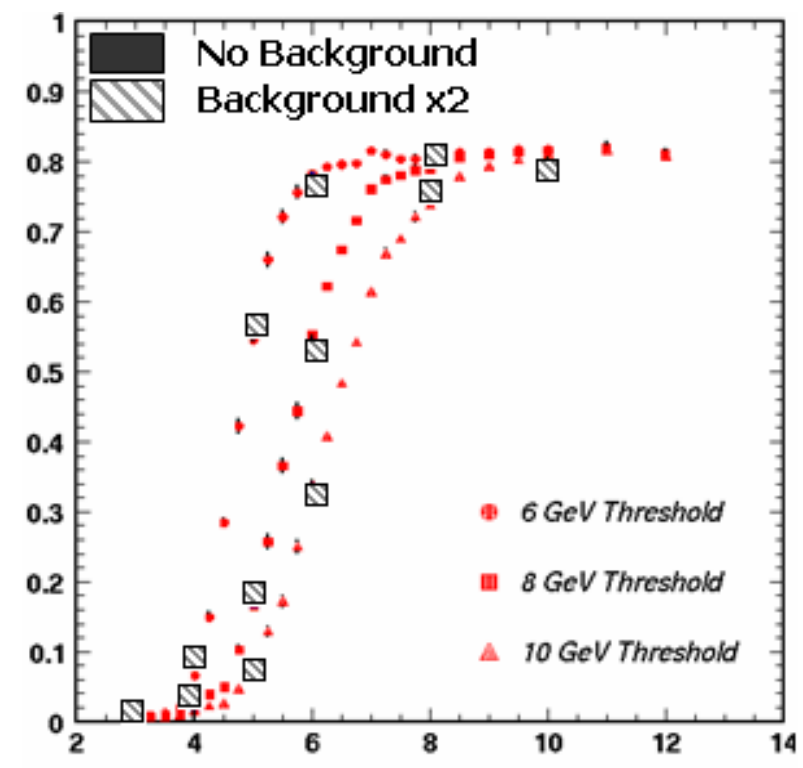

Figure 9: effect of background on low $p_{\mathrm{T}}$ trigger efficiency

\section{CONCLUSIONS}

RPC detector are used in ATLAS as trigger detectors for the barrel muon spectrometer. Their high time resolution of $1.5 \mathrm{~ns}$ and good spatial resolution of about $1 \mathrm{~cm}$ allow fast detection of candidate muons coming from Interaction Point and having transverse momentum typical of interesting events. RPCs, built in number of more than 1200 units, are subject to several and severe quality tests, from production to assembly, to integration on MDT and finally cosmic ray certification. Muon trigger is based on Coincidence Matrix whose contents has been calculated and checked with simulation in this work, together with other important trigger parameters. Efficiency, trigger rates and background effects have been estimated too. Integrated packages of MDT and RPC are currently being installing in the ATLAS pit, where on site cosmic rays test will be started on May 2006 in order to proceed to the final commissioning of the Muon Spectrometer.

1. The ATLAS Collaboration. Level-1 Trigger Technical Design Report. CERN TDR-12, June 1998

2. G. Carlino et al. The RPC LVL1 Trigger System of the muon Spectrometer of the ATLAS experiment at LHC. IEEE Trans.Nucl.Sci.51:1581, 2004, Portland.

3. V. Bocci et. al. The Coincidence Matrix ASIC of the Level-1 Muon Barrel Trigger of the ATLAS Experiment. IEEE Transaction on Nuclear Science, vol. 50 n. 4, 1078 (Aug. 2003)

4. The ATLAS Collaboration. First level trigger Technical Design Report. CERN/LHCC/98-14 pg. 247

5. M. Alviggi et al. First results of the cosmic rays test of RPC of the ATLAS muon spectrometer at LHC. Nucl. Instr. and Meth. A 518 (2004) 79.

6. G. Aielli et al. Test and performance of the RPC trigger chambers of the ATLAS experiment at LHC. Nucl. Instr. and Meth. A 533 (2004) 193.

7. G. Chiodini et al. ATLAS RPC Cosmic Ray Teststand in Lecce. Proceeding of XXIV Physics in Collision - Boston - June 27-29, 2004, e-print archive: Physics/0409075

8. A. Aloisio et al. The Trigger Chambers of the ATLAS muon spectrometer: production and tests. NIM-A 535 (2004) 265-271 
${ }^{9}$ ATHENA: the ATLAS Common Frameworkdeveloper guide. Version 8.0 (Feb. 2004). Available at: http://ATLAS.web.cern.ch/ATLAS/GROUPS/SOFTW ARE/OO/architecture/General/Documentation/Athena DeveloperGuide-8.0.0-draft.pdf

10. T. Sjostrand, PYTHIA 5.7 and JETSET. CERN-TH.7112/93, 1993

11. J.Ranft, DPMJET version H3 and H4. INFN-AE-97-45 